\title{
Evaluation of combined famotidine with quercetin for the treatment of peptic ulcer: in vivo animal study
}

This article was published in the following Dove Press journal:

Drug Design, Development and Therapy

I 3 April 2015

Number of times this article has been viewed

\author{
Mohammed AS Abourehab ${ }^{1,2}$ \\ Khaled A Khaled' \\ Hatem AA Sarhan' \\ Osama AA Ahmed ${ }^{1,3}$ \\ 'Department of Pharmaceutics \\ and Industrial Pharmacy, Faculty \\ of Pharmacy, Minia University, Minia, \\ Egypt; ${ }^{2}$ Department of Pharmaceutics, \\ Faculty of Pharmacy, Umm Al-Qura \\ University, Makkah, Saudi Arabia; \\ ${ }^{3}$ Department of Pharmaceutics \\ and Industrial Pharmacy, Faculty \\ of Pharmacy, King Abdulaziz \\ University, Jeddah, Saudi Arabia
}

Abstract: The aim of this work was to prepare a combined drug dosage form of famotidine (FAM) and quercetin (QRT) to augment treatment of gastric ulcer. FAM was prepared as freeze-dried floating alginate beads using ion gelation method and then coated with Eudragit RL100 to sustain FAM release. QRT was prepared as solid dispersion with polyvinyl pyrrolidone K30 to improve its solubility. Photo images and scanning electron microscope images of the prepared beads were carried out to detect floating behavior and to reveal surface and core shape of the prepared beads. Anti-ulcerogenic effect and histopathological examination of gastric tissues were carried out to investigate the effect of the combined drug formulation compared with commercial FAM tablets and FAM beads. Gastric glutathione (GSH), superoxide dismutase, catalase, tissue myeloperoxidase, and lipid peroxidation enzyme activities and levels in rat stomach tissues were also determined. Results revealed that spherical beads were formed with an average diameter of $1.64 \pm 0.33 \mathrm{~mm}$. They floated immediately with no lag time before floating, and remained buoyant throughout the test period. Treatment with a combination of FAM beads plus QRT showed the absence of any signs of inflammation or hemorrhage, and significantly prevented the indomethacin-induced decrease in GSH levels $(P<0.05)$ with regain of normal GSH gastric tissue levels. Also, there was a significant difference in the decrease of malondialdehyde level compared to FAM commercial tablets or beads alone $(P<0.05)$. The combined formula significantly improved the myeloperoxidase level compared to both the disease control group and commercial FAM tablet-treated group $(P<0.05)$. Formulation of FAM as floating beads in combination with solid dispersion of QRT improved the anti-ulcer activity compared to commercially available tablets, which reveals a promising application for treatment of peptic ulcer.

Keywords: anti-ulcerogenic effect, biochemical studies, floating beads, histopathological examination

\section{Introduction}

Peptic ulcer, which includes both gastric and duodenal ulcer, is one of the most common disorders affecting the gastrointestinal system. ${ }^{1}$ The pathophysiology of acid-peptic disease is attributed mainly to the imbalance between aggressive factors (such as acid, pepsin, Helicobacter pylori infection, smoking, stress, and excessive alcohol intakes) and cytoprotective action of the gastrointestinal mucosa, like secretion of bicarbonate, mucus, and prostaglandins. ${ }^{2}$ Histamine is a major stimulant for acid secretion through the $\mathrm{H}_{2}$ receptors; therefore, blocking these receptors may lead to reduction in acid secretion. The inhibition of gastric acid secretion is still a key therapeutic target for the ulcer diseases of any cause, gastro-esophageal reflux disease, Zollinger-Ellison syndrome, and gastritis. ${ }^{3}$ This goal is best achieved by blocking the acid secretory effect of histamine through the use of $\mathrm{H}_{2}$
Correspondence: Osama AA Ahmed Department of Pharmaceutics and Industrial Pharmacy, Faculty of Pharmacy, King Abdulaziz University, PO Box 80200 , Jeddah 21589, Saudi Arabia

Tel +96626400000, ext 2225।

Email oaahmed@kau.edu.sa 
receptor antagonists or the irreversible $\mathrm{H}^{+} / \mathrm{K}^{+}$ATPase inhibitors, popularly referred to as proton pump inhibitors. ${ }^{4} \mathrm{H}_{2}$ receptor antagonist are specific antagonists that inhibit acid secretion by competitively and reversibly blocking the $\mathrm{H}_{2}$ receptors on the basolateral membrane of the parietal cell. ${ }^{5}$

Famotidine (FAM) is the most potent antagonist available for clinical use. ${ }^{6}$ FAM is used for treatment of gastroesophageal reflux, heart burn, and peptic ulcers without side effects. ${ }^{7}$ Studies showed that the concomitant use of FAM could increase the cure rates of $H$. pylori infection by a triple therapy with lansoprazole, clarithromycin, and amoxicillin at the standard doses. ${ }^{8}$ FAM is incompletely absorbed from the gastrointestinal tract. The low oral bioavailability (40\%-45\%) and short biological half-life (2.5-3.5 hours) of FAM favors development of a sustained release formulation. ${ }^{9}$ FAM has pH-dependent solubility (basic drug, pKa 7.06); therefore, its gastric retention would allow adequate time for its dissolution, the rate limiting step in drug absorption.

Flavonoids are intensively studied because of their proposed potentially beneficial effects in preventing various diseases. ${ }^{10-12}$ The flavonoid quercetin (QRT) is reported to exhibit an antiradical property toward hydroxyl and peroxyl radicals and superoxides anions, a mechanism involved in peptic ulcer. ${ }^{13}$ It was demonstrated that QRT is an effective cytotoxic agent in the case of gastric carcinoma cell lines. ${ }^{14} \mathrm{QRT}$ has been shown to have anti-ulcer and gastroprotective effects. ${ }^{15,16}$ QRT inhibits growth of $H$. pylori in a dose-dependent manner in vitro, which contributes to its anti-ulcer effect. ${ }^{17}$

Floating drug delivery systems are among the mechanisms available for controlling the gastric retention of solid dosage forms. A gastro-retentive floating system made up of multiple-unit particulate (eg, beads) has relative merits compared with a single-unit preparation. Floating calcium alginate beads have been investigated as a possible gastroretentive dosage form, and are designed to enhance the bioavailability of certain drugs from oral preparations. ${ }^{18}$

This study is aimed at the preparation of a combined oral dosage form containing floating FAM alginate beads and a solid dispersion of QRT-polyvinyl pyrrolidone K30 (PVP k30) to augment their effect for treatment of peptic ulcer. The anti-ulcerogenic activity of FAM alginate floating beads in combination with QRT-PVP was evaluated using rats as an animal model. Histopathological examinations of gastric tissues were carried out to investigate the effect of the prepared formulation compared with commercial FAM product and FAM beads only. Gastric glutathione (GSH), superoxide dismutase (SOD), catalase (CAT), tissue myeloperoxidase (MPO), and lipid peroxidation (LPO) enzyme activities and levels in rat stomach tissues were also determined.

\section{Materials and methods}

FAM was a kind gift from Medical Union Pharmaceutical (MUP, Abou Sultan - Ismailia, Egypt) Company, Egypt. Eudragit RL100 was a kind gift from Evonik Rohme, Germany. Sodium alginate, medium viscosity (200 cPs for $1 \%$ aqueous solution at $20^{\circ} \mathrm{C}$ ) was purchased from Alpha Laboratories Ltd., Eastleigh, Hampshire, UK. PVP $\mathrm{k} 30$, anhydrous calcium chloride, sodium dihydrogen phosphate, sodium hydrogen phosphate dibasic, and indomethacin were from Sigma-Aldrich Co., St Louis, MO, USA.

\section{Methods}

\section{Preparation and characterization of coated FAM floating beads}

Floating freeze-dried calcium alginate beads were prepared by the ionotropic gelation method. Sodium alginate was dissolved in deionized water to prepare solutions of $2 \%$ $(\mathrm{w} / \mathrm{v})$. FAM was dispersed in sodium alginate solution at a drug:polymer ratio of 2:1 w/w, and the mixture was homogenized (Ultra Turrax T25, IKA ${ }^{\circledR}$-Werke GmbH \& Co. KG, Staufen, Germany) for 15 minutes. The bubblefree drug-loaded mixture was extruded through a $22 \mathrm{G}$ syringe needle, at a constant rate, into $250 \mathrm{~mL}$ of $0.5 \mathrm{M}$ calcium chloride solution, maintained under gentle agitation at a dropping distance of $5 \mathrm{~cm}$ from the surface of calcium chloride solution. The formed beads were cured for 30 minutes at room temperature, separated, washed three times with $500 \mathrm{~mL}$ of distilled water, then collected and freeze-dried (Christ Beta 1-8 LD Freeze Dryer; Martin Christ Gefriertrocknungsanlagen $\mathrm{GmbH}$, Osterode am Harz, Germany). The prepared beads were then coated by immersion in Eudragit RL100 (5\% w/v) in absolute ethanol followed by solvent evaporation using a rotary evaporator (Rotavapor II; BÜCHI Labortechnik AG, Flawil, Switzerland). The coating process continued until coating weight was obtained $(5 \% \mathrm{w} / \mathrm{w})$. The coated beads were then dried at room temperature for 24 hours. The dried beads were stored in desiccator containing anhydrous calcium chloride. Photo images and scanning electron microscope images of the prepared beads were carried out to detect floating behavior and to reveal the surface and core shape of the prepared beads.

\section{Preparation of QRT solid dispersion}

Weighed amounts of QRT and PVP K30, to prepare a 1:1 (w/w) ratio, were dissolved in absolute ethanol and coprecipitated by slow evaporation of the solvent at $60^{\circ} \mathrm{C}$ using rotary evaporator. The produced solid mass was further 
dried in an oven at $70^{\circ} \mathrm{C}$ for 24 hours. The dried solid mass was pulverized and sieved to obtain a particle size range of 125-250 $\mu \mathrm{m}$ and kept in a desiccator.

It is worth mentioning that the detailed investigation of the factors affecting characteristics of the prepared beads using different methods, in addition to various parameters that influence the behavior of the beads in vitro and QRT preparation, are to be submitted as a separate article.

\section{In vivo studies}

\section{Animals}

Male albino rats (150-250 g) were used. Animals were supplied from the Animal House of Faculty of Medicine, Assiut University, Assiut, Egypt. Animals were kept for 1 week before the study as a pre-breeding period for acclimatization to accommodate the test conditions. Animal use was approved by the Institutional Review Board for Animal Research/Studies who ensured that the care and use of animals conformed to the EU Directive $2010 / 63 /$ EU on the protection of animals used for scientific purposes.

\section{Anti-ulcerogenic effect}

Animals were fasted for 24 hours with free access to water. Gastric ulceration was induced by oral administration of aqueous suspension of indomethacin in $1 \%$ sodium carboxy-methylcellulose (CMC) as $50 \mathrm{mg} / \mathrm{kg}$ of body weight, $2 \mathrm{~mL}$ orally, for all groups except animals in group 1 for 2 consecutive days. The animals were further fasted for 12 hours, and then randomly divided into five experimental groups of eight rats each. The first and second groups received $1 \%$ sodium $\mathrm{CMC}$ solution ( $2 \mathrm{~mL}$, oral) once daily for 15 consecutive days and represent control and disease control, respectively. The third group (standard) received crushed commercial FAM tablets. Group 4 (test 1 group), received the prepared FAM alginate beads. Group 5 (test 2 group) received FAM alginate beads and QRT-PVP coprecipitate.

FAM and QRT doses were $12 \mathrm{mg} / \mathrm{kg}$ and $50 \mathrm{mg} / \mathrm{kg}$, respectively. They were administered as a suspension in $1 \%$ sodium CMC ( $2 \mathrm{~mL}$, oral) once daily for 15 consecutive days. On the 16th day, excised stomachs were examined macroscopically for hemorrhagic lesions. The number of ulcers per stomach was noted and the severity of the ulcers was observed microscopically. Ulcer index of each animal was calculated by the following scoring system: 0 for normalcolored stomach, 0.5 for red coloration, 1 for spot ulceration, 1.5 for hemorrhagic streaks, 2 for ulcers $>3 \mathrm{~mm}$ but $<5 \mathrm{~mm}$, 3 for ulcer $>5 \mathrm{~mm}$, and 4 for perforations.

\section{Histopathological examination of gastric tissues}

Specimens of the isolated gastric tissues were fixed in $10 \%$ formalin solution for 24 hours. Sections of 4-5 $\mu \mathrm{m}$ thickness were stained with hematoxylin and eosin. The sections were mounted and observed under light microscope.

\section{Biochemical studies}

GSH, SOD, CAT, MPO, and LPO enzyme activities and levels in rat stomach tissues were determined. For biochemical estimations, $0.5 \mathrm{~g}$ of the whole gastric tissue samples were ground with liquid nitrogen and then mixed with the appropriate buffer. Mixtures were homogenized on ice. Homogenates were used for determination of the enzymatic activities. ${ }^{19}$

GSH determination was carried out using a modified Sedlak and Lindsay method. ${ }^{20}$ Briefly, the mucosal surface of the stomach was collected by scraping, weighed, and properly homogenized in $2 \mathrm{~mL}$ of $50 \mathrm{mM}$ Tris-HCl buffer containing $20 \mathrm{mM}$ ethylenediaminetetraacetic acid and $0.2 \mathrm{mM}$ sucrose, $\mathrm{pH}$ 7.5. The homogenate was immediately precipitated with $0.1 \mathrm{~mL}$ of ice-cold $25 \%$ trichloroacetic acid and centrifuged at 4,000 rpm for 40 minutes at $4{ }^{\circ} \mathrm{C}$. The supernatant was measured at wavelength of maximum absorption $\left(\lambda_{\max }\right) 412 \mathrm{~nm}$. LPO content was estimated by measuring the formation of malondialdehyde (MDA) - an end product of LPO - using the thiobarbituric acid test. The corpus mucosa was scraped, weighed, and homogenized in $10 \mathrm{~mL}$ of $100 \mathrm{~g} / \mathrm{L} \mathrm{KCl}$. The homogenate $(0.5 \mathrm{~mL})$ was added to a solution containing $0.2 \mathrm{~mL}$ of $80 \mathrm{~g} / \mathrm{L}$ sodium lauryl sulfate, $1.5 \mathrm{~mL}$ of $200 \mathrm{~g} / \mathrm{L}$ acetic acid, $1.5 \mathrm{~mL}$ of $8 \mathrm{~g} / \mathrm{L}$ 2-thiobarbiturate, and $0.3 \mathrm{~mL}$ of distilled water. The mixture was incubated at $98^{\circ} \mathrm{C}$ for 1 hour. Upon cooling, $5 \mathrm{~mL}$ of n-butanol:pyridine (15:1 v/v) was added. The mixture was vortexed for 1 minute and centrifuged at 4,000 rpm for 30 minutes. The supernatant was measured spectroscopically at $\lambda_{\text {max }}$ of $532 \mathrm{~nm}$ for MDA. ${ }^{21}$ MPO activity was determined using Bradley procedure. ${ }^{22}$ SOD activity was measured using the method of Sun et al. ${ }^{23}$ CAT measurement was carried out by estimation of $\mathrm{H}_{2} \mathrm{O}_{2}$ decomposition in the presence of CAT at $\lambda_{\max } 240 \mathrm{~nm}^{22}$

\section{Statistical analysis}

The experimental data were expressed as mean \pm standard deviation. Statistical analysis was carried out using GraphPad Prism version 3.0 (GraphPad Software, Inc., La Jolla, CA, USA). Groups of data were compared with one-way analysis of variance, followed by the Tukey's test. The values were considered statistically significant when $P<0.05$. 


\section{Results and discussion Preparation of FAM floating beads}

Sodium alginate can form gel by ionotropic gelation with divalent cations such as $\mathrm{Ca}^{2+}$ and $\mathrm{Ba}^{2+}$. Cross-links are formed between the divalent ions and the negatively charged carboxyl groups of the alginate molecules. ${ }^{24}$ Alginate beads are one of the particulate delivery systems that protect drugs from the acidic environment, and improve bioavailability of the target drug at a specific site. ${ }^{25}$ Spherical beads were formed with an average diameter of $1.64 \pm 0.33 \mathrm{~mm}$. They floated immediately with no lag time float, and remained buoyant throughout the test period (Figure 1). This may be due to their highly porous internal structure as shown in scanning electron microscope images (Figure 2). This might be as a result of water sublimation leaving pores and cavities in the alginate matrix.

\section{Preparation of QRT solid dispersion}

Despite the biological activities of QRT, its low aqueous solubility hampers its use as a therapeutic agent. ${ }^{26}$ The solubility of QRT determined at room temperature was $0.441 \pm 0.0487 \mu \mathrm{g} / \mathrm{mL} .{ }^{27}$ Therefore, any attempt to enhance the dissolution rate would improve absorption and bioavailability of QRT. ${ }^{28}$ Different methods and techniques had been utilized to improve the solubility of QRT. ${ }^{29-33}$ Complexation of QRT with PVP could be an applicable approach to improve QRT dissolution, and hence bioavailability. PVP improves markedly the aqueous dissolution of QRT when incorporated with the later as coprecipitate. Solid dispersions of QRT containing high proportions of PVP were most effective in enhancing the drug dissolution rate (full detailed data will be published elsewhere).

\section{In vivo studies}

Nonsteroidal anti-inflammatory drugs (NSAIDs) may damage the gastrointestinal mucosa by two distinct mechanisms:
1) suppression of prostaglandin synthesis and 2) direct irritant action causing alterations of mucosal permeability. ${ }^{34}$ In the stomach, prostaglandins play a vital protective role by stimulating secretion of $\mathrm{HCO}_{3}-$ and mucous, maintaining mucosal blood flow, and regulating mucosal cell turnover and repair. Thus, the suppression of prostaglandin synthesis by NSAIDs like indomethacin results in increased susceptibility to mucosal injury and gastroduodenal ulceration..$^{35}$ Previous results have shown that reactive oxygen species (ROS) plays an important role in pathogenesis of mucosal damage caused by indomethacin besides inhibition of cyclooxygenase enzymes. ${ }^{36}$ Indomethacin induces higher gastric damage in rats when compared with other NSAIDs.$^{37}$ Additionally, It is reported that FAM prevents indomethacin-induced gastric ulcers in rats. ${ }^{38}$

Macroscopic examination of the investigated rat stomachs is shown in Figure 3. The normal control group (1) stomachs (Figure 3A) revealed normal mucosa, no inflammation, and no hemorrhage or patches of hyperemia. On the other hand, the diseased rats group (2) showed marked severe injuries in the gastric mucosa (Figure 3B). Oral administration of indomethacin $(50 \mathrm{mg} / \mathrm{kg})$ produced severe hemorrhagic lesions predominantly on glandular mucosa segment of the stomach and few or none in the antrum. Dark reddish patches of ulcers covered the majority of the stomach body, and there were numerous ulcer patches with different forms and sizes within the stomach mucosa. There was remarkable hyperemia in the stomach mucosa. Upon treating the animals with crushed commercial FAM tablets (Figure 3C), the stomachs showed significantly lower numbers of ulcers. They showed moderate mucosal injuries compared to those of ulcerated control rats, and ulcers appeared as discontinuous lesions in the gastric mucosa; no perforation was noticed in all treated animal groups. While treatment with FAM beads (Figure 3D) showed milder ulceration reaction, few small lesions appeared in the gastric mucosa and produced more prominent inhibition
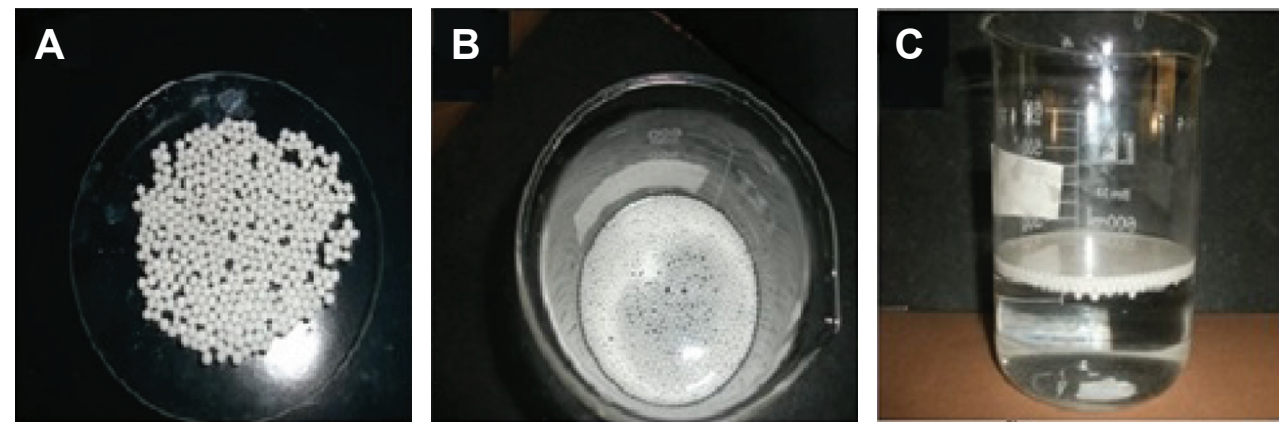

Figure I Photograph of the prepared freeze-dried famotidine-loaded alginate beads.

Notes: (A) Dried beads; (B) dried beads floated in simulated gastric fluid (top view); (C) dried beads floating in simulated gastric fluid (side view). 

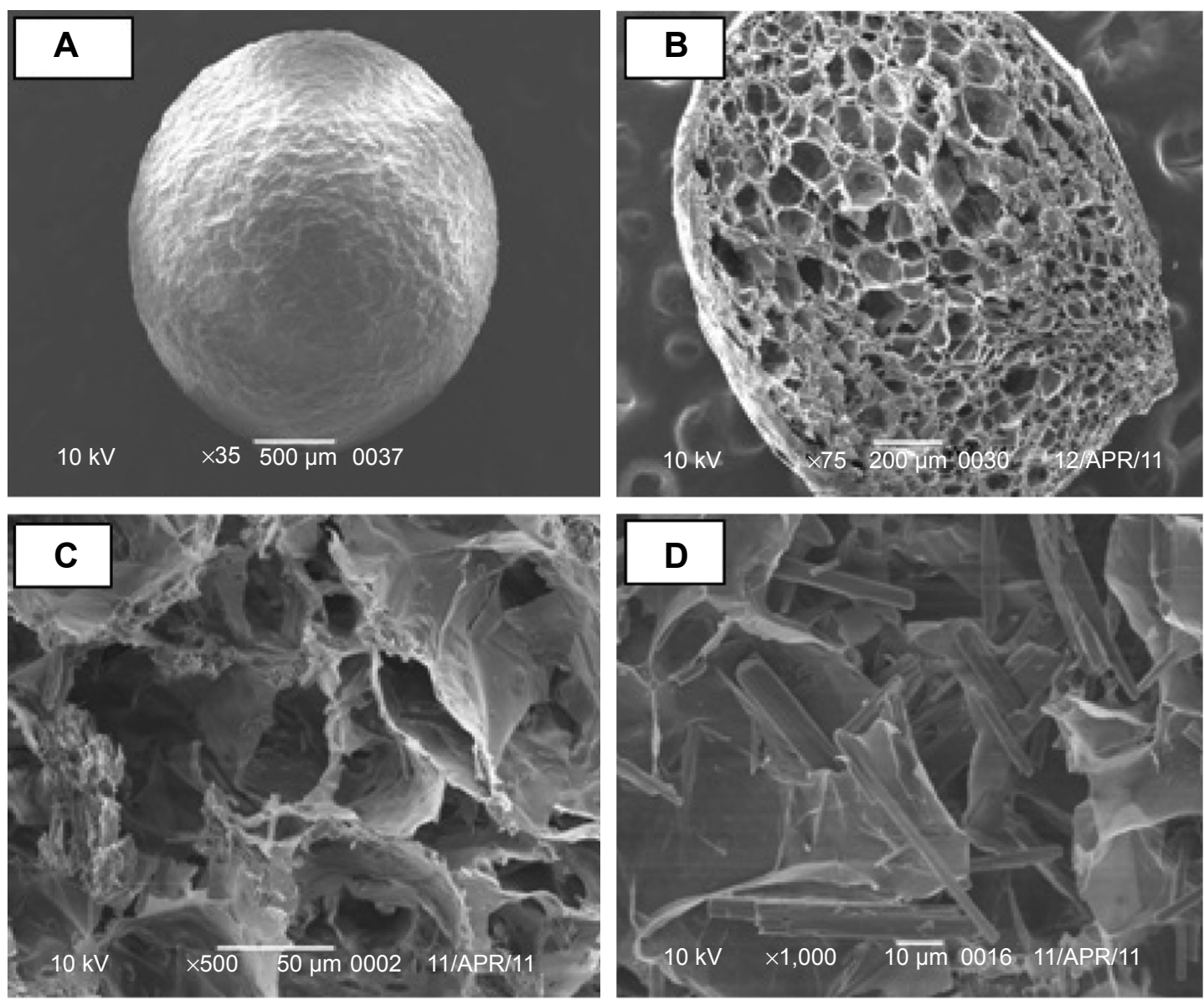

Figure 2 Scanning electron microscope micrographs of freeze-dried calcium alginate beads.

Notes: (A) External surface; (B) internal cross-section with magnification power $\times 75$; (C) internal cross-section with magnification power $\times 500$; (D) internal cross-section with magnification power $\times 1,000$.

effect when compared with the commercial FAM tablets treatment group. On the other hand, treatment with a combination of beads formula plus QRT-PVP coprecipitate (Figure 3E) showed a nearly normal stomach appearance, as minor ulceration was observed in addition to the absence of any sign of inflammation or hemorrhage. Animals of treated groups were healthy and showed nearly normal activity during the experiment.

Figure 4 illustrates ulcer indices for animals in the indomethacin-induced ulcerated model. Ulcer index in group 2 was $42.50 \pm 0.83$, which confirms ulcer formation. The ulcer index for the rats treated with FAM tablets was $30.53 \pm 0.86$; a value significantly lower than that of group $2(P<0.05)$. On the other hand, the ulcer index for rats treated with FAM beads was $17.58 \pm 0.57$ which is significantly reduced compared to the disease control group (2) and the animals treated with commercial FAM tablets. However, rats treated with formulated FAM beads in combination with QRT-PVP showed an ulcer index of $6.46 \pm 0.64$, which was significantly the lowest compared with all other ulcer indices of investigated groups $(P<0.05)$ as shown in Figure 4.

It is clear from the obtained results that treatment of animals with the formulated beads produced more effective protection against gastric ulcers when compared with that of the commercial tablets. This may be explained on the basis of the extended duration of drug release and providing effective drug concentration over a prolonged time. Upon using combination treatment of FAM beads plus QRT-PVP, the data showed a significantly improved protection against gastric ulceration when compared with FAM beads alone. This could be attributed to the gastroprotective effect of QRT as a result of its antioxidation and free radical-scavenging property, which inhibits lipid peroxide level in gastric mucosa. ${ }^{39}$

\section{Histological examination}

Histopathological examination was carried out to confirm the morphological changes in stomach tissues in the studied animal groups. Figure 5 shows the histopathological effect of different treatments on indomethacin-induced ulcer model. 

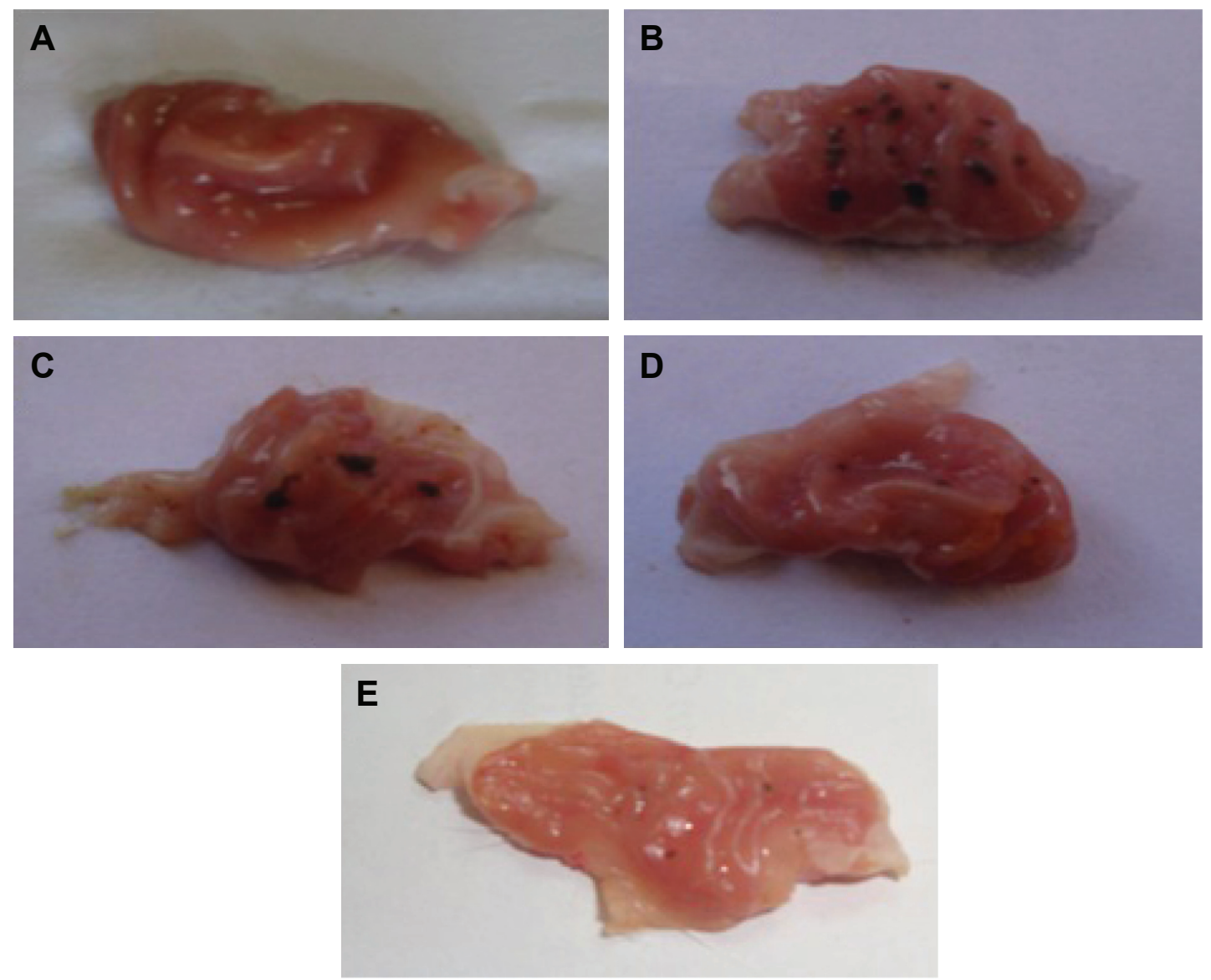

Figure 3 Gross appearance of the gastric mucosa in stomachs of indomethacin-induced models.

Notes: (A) Normal control; (B) disease control group; (C) commercial FAM; (D) FAM beads; (E) FAM beads plus QRT-PVP.

Abbreviations: FAM, famotidine; PVP, polyvinyl pyrrolidone; QRT, quercetin.

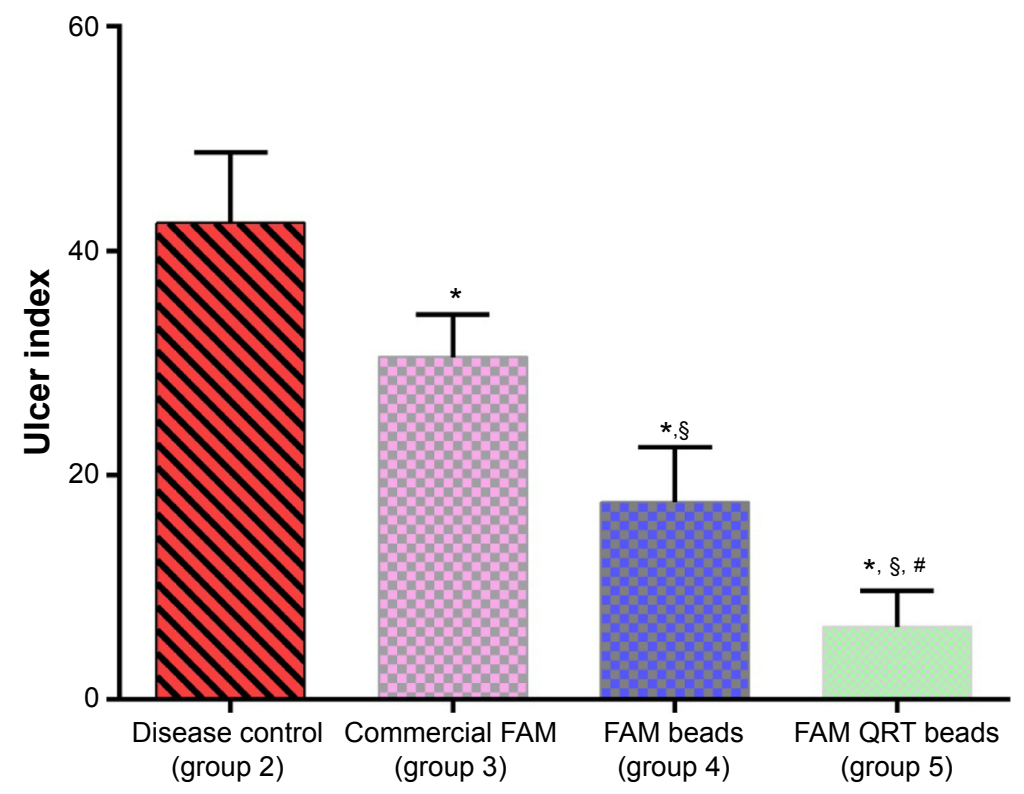

Figure 4 Effects of different treatments on gastric ulcer in indomethacin-induced ulcers in rats.

Notes: All values are expressed as mean \pm standard deviation; $n=8$ animals in each group. *Significant difference versus indomethacin-treated group (group 2 ); ${ }^{\S}$ significant difference versus commercial famotidine group (group 3); " significant difference versus famotidine beads-treated group (group 4 ). $P<0.05$.

Abbreviations: FAM, famotidine; QRT, quercetin. 

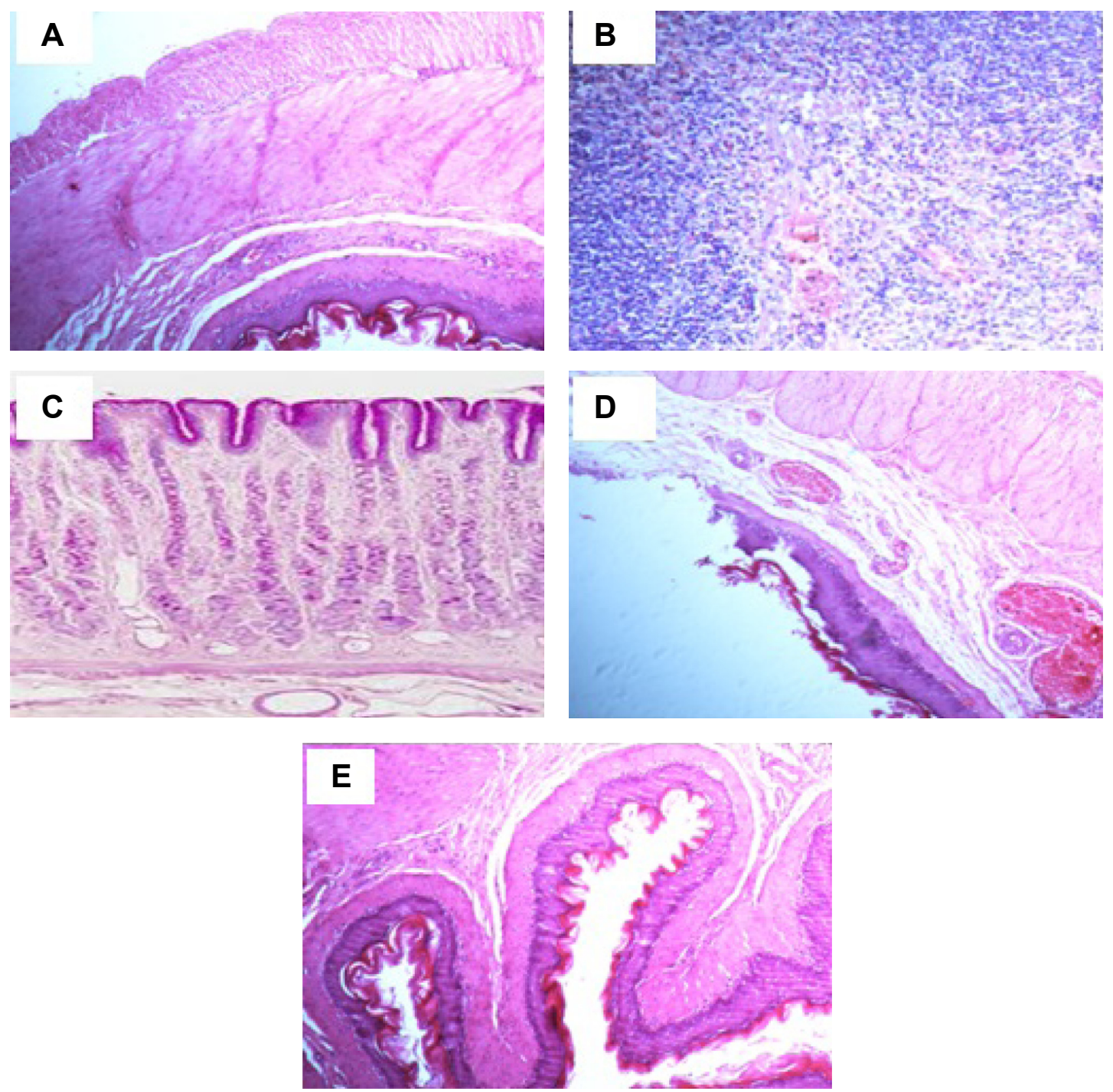

Figure 5 Histological evaluation of stomach tissues from indomethacin-induced ulcer model in rats of hematoxylin and eosin staining of gastric mucosa (magnification $\times 100)$. Notes: (A) Normal control; (B) disease control group; (C) commercial FAM; (D) FAM beads; (E) FAM beads plus QRT-PVP.

Abbreviations: FAM, famotidine; PVP, polyvinyl pyrrolidone; QRT, quercetin.

Stomach histology of control normal rats shows normal microscopic architecture with normal glands, without any signs of abnormalities. The parietal cells located in the upper half of gastric glands with eccentric nuclei and pale eosinophilic vacuoles in the cytoplasm (Figure 5A). On the other hand, the figure reveals a severe inflammatory reaction, manifested by submucosal edema with local mononuclear leucocytic infiltration (mainly lymphocytes and few eosinophils) in the lamina propria, muscularis mucosa, and submucosal layers of the stomach tissue for the indomethacin-treated control group (2). The submucosal blood vessels were congested and the muscular coat was hypertrophied and partially hyalinized. These observations revealed the acute phase of ulcer and a severe inflammatory reaction (Figure 5B). The stomach tissue of group 3, receiving the commercial FAM tablets, showed moderate inflammatory reaction (Figure 5C). The reaction severity was milder in case of animals receiving the beads (Figure 5D), who showed moderate amounts of leucocytic inflammatory cell infiltration in the submucosal layer and muscularis mucosa. Animals in group 5, receiving the beads formula plus the QRT-PVP (Figure 5E), were the best-preserved group in terms of morphological integrity. It showed a uniform epithelial and glandular structure and arrangement, and good tissue architecture. Only minor leucocytic inflammatory cells were encountered in tissue sections.

\section{Biochemical studies}

Gastric ulcer production by indomethacin is mainly attributed to the inhibition of prostaglandin synthesis in the 
stomach tissue. It has also been shown that ROS plays an important role in the pathogenesis of mucosal damage caused by indomethacin. ${ }^{40,41}$ The results revealed elevated levels of MPO and MDA in the gastric tissue of the animals that were given indomethacin. On the other hand, enzymatic and nonenzymatic antioxidant parameters, such as GSH, SOD, and CAT, were reduced. ${ }^{42}$ These antioxidants are protective factors in the control of indomethacin-induced damage. GSH, SOD, and CAT prevent the tissue damage by maintaining ROS at low levels in the cells. ROS-mediated degradation of cell membrane initiates mucosal lesions, increased vascular permeability, and depletion of the mucus layer. ${ }^{43}$

SOD is the antioxidant enzyme which catalyzes the conversion of superoxide free radical $\left(\mathrm{O}_{2}^{-}\right)$to hydrogen peroxide $\left(\mathrm{H}_{2} \mathrm{O}_{2}\right)$ and to molecular oxygen $\left(\mathrm{O}_{2}\right)$. SOD and endogenous antioxidant enzymes render the free radicals harmless and protect the tissues from the harmful effects of free radicals and active oxygen species. When these antioxidants' defense mechanisms are inadequate, free radicals lead to serious damage in the tissues, with LPO being the most important and most harmful effect that the free radicals trigger in the cell. ${ }^{44}$

MDA is the end product of LPO which leads to further damage in the cells, whereas MPO is the enzyme that catalyzes the production of toxic hypochlorous acid from $\mathrm{H}_{2} \mathrm{O}_{2}$. It is present in the phagocytic cells. Excessive production of MPO and other reactive radicals causes oxidative damage. ${ }^{45}$ A previous report demonstrated that MPO activity increases in NSAID-damaged stomach tissue. ${ }^{37} \mathrm{CAT}$ is a highly reactive enzyme that detoxifies hydrogen peroxide and disrupts free radical synthesis. CAT reacts with $\mathrm{H}_{2} \mathrm{O}_{2}$ to form water and molecular oxygen. ${ }^{46}$ Indomethacin-induced gastric ulcers may be eliminated with antioxidant effect. It was demonstrated that the increase of mucosal oxidants and the decrease of enzymatic and nonenzymatic antioxidants, induced by indomethacin, may be reversed with the use of FAM ${ }^{47}$ Within the flavonoid family, QRT is the most potent scavenger of ROS. ${ }^{48}$ Therefore, a combination of QRT and
FAM could improve anti-ulcer activity. Effects of different treatments on the levels of GSH and various enzymes are shown in Table 1.

\section{Gastric GSH levels}

The elevated levels of GSH in gastric mucosa are essential to maintain mucosal integrity, and depletion of GSH could induce mucosal ulceration. In addition, depletion of GSH could limit the activity of GSH-dependent enzymes. ${ }^{49}$ Table 1 shows that the GSH level in the gastric tissues of rats given indomethacin was significantly lower than that of the normal control group rats $(P<0.05)$. Treatment of animals with FAM increased the total GSH level significantly when it was compared with diseased control group (group 2) $(P<0.05)$. Treatment with FAM beads (group 4) significantly improved the GSH level in the stomach tissue when it was compared with group $3(P<0.05)$. Treatment with a combination of FAM beads plus QRT significantly prevented the indomethacin-induced decrease in GSH levels $(P<0.05)$ and the gastric tissue regained its normal GSH levels.

\section{Gastric LPO levels}

From the data of Table 1, the MDA level of indomethacintreated (group 2) increased significantly when compared with the normal control rat group. MDA level was not significantly $(P>0.05)$ decreased compared to the diseased control group in rats treated with commercial FAM tablets, whereas the level of MDA decreased significantly with the use of FAM beads $(P<0.05)$. Upon using combination treatment, there was a significant difference in the decrease of MDA level compared to FAM commercial tablets or beads alone $(P<0.05)$. LPO was almost completely inhibited by FAM beads plus QRT; the difference between the normal control and QRT group was not significant $(P>0.05)$ as the value of the LPO may be normalized. It is clear from the results that there was a significant positive correlation between the ulcer index values and LPO, indicating that LPO may be one of the main causes of mucosal damage.

Table I Effects of different treatments on the level of GSH and various enzymes in different experimental groups

\begin{tabular}{llllll}
\hline Group & $\begin{array}{l}\text { GSH } \\
(\mathbf{n M} / \mathbf{m g})\end{array}$ & $\begin{array}{l}\text { LPO } \\
(\text { MDA level } \mathbf{n M} / \mathbf{g})\end{array}$ & $\begin{array}{l}\text { MPO } \\
(\mu \mathrm{M} / \mathbf{m i n} / \mathbf{m g})\end{array}$ & $\begin{array}{l}\text { SOD } \\
(\mathbf{m M} / \mathbf{m i n} / \mathbf{m g})\end{array}$ & $\begin{array}{l}\text { CAT } \\
(\mathbf{m M} / \mathbf{m i n} / \mathbf{m g})\end{array}$ \\
\hline 1 & $4.65 \pm 0.08$ & $21.81 \pm 0.35$ & $41.55 \pm 0.62$ & $47.76 \pm 0.86$ & $232.40 \pm 2.88$ \\
2 & $1.06 \pm 0.03$ & $48.56 \pm 0.81$ & $74.06 \pm 0.66$ & $22.92 \pm 0.64$ & $103.40 \pm 2.13$ \\
3 & $2.43 \pm 0.02$ & $37.36 \pm 0.82$ & $59.46 \pm 0.46$ & $30.70 \pm 0.68$ & $166.55 \pm 3.47$ \\
4 & $3.48 \pm 0.03$ & $27.43 \pm 0.68$ & $48.21 \pm 0.57$ & $39.45 \pm 0.71$ & $206.58 \pm 4.16$ \\
5 & $4.11 \pm 0.07$ & $22.28 \pm 0.34$ & $42.43 \pm 0.59$ & $45.79 \pm 0.39$ & $225.28 \pm 2.95$ \\
\hline
\end{tabular}

Note: Data presented as mean \pm standard deviation.

Abbreviations: CAT, catalase; GSH, gastric glutathione; LPO, lipid peroxidation; MPO, tissue myeloperoxidase; MDA, malondialdehyde; SOD, superoxide dismutase. 


\section{Gastric MPO activity}

The MPO activity is an index of neutrophil-dependent inflammatory response and neutrophil infiltration in various gastric injuries..$^{50} \mathrm{MPO}$ activity is a sensitive and specific marker of acute inflammation and reflects polymorphonuclear cell infiltration into the parenchyma. ${ }^{46} \mathrm{MPO}$ is an essential marker for normal neutrophil function. Table 1 shows the effect of different treatments on the MPO activity. From Table 1 , it is clear that indomethacin significantly increased MPO levels in rat stomach tissue compared to the normal control rats $(P<0.05)$.

The interaction of MPO with radicals such as $\mathrm{O}_{2}^{-}, \mathrm{H}_{2} \mathrm{O}_{2}$, and $\mathrm{OH}^{-}$released as a result neutrophil activation produces hypochlorous acid and $\mathrm{N}$-chloramine that lead to tissue damage. ${ }^{51}$ Indomethacin exerts its effects via inhibition of the MPO pathways. ${ }^{52}$ Treatment of rats with commercial FAM tablets produced a nonsignificant increase in the MPO level $(P>0.05)$, while treatment of rats with FAM beads significantly improved the MPO level compared to both the disease control group and commercial FAM tablet-treated group $(P<0.05)$. Treatment of rats with a combination of FAM beads plus QRT revealed significant improvement in the MPO level, as compared to beads alone $(P<0.05)$. In addition, treatment of rats with the combination of beads and QRT normalized the MPO level as there was nonsignificant difference between group 5 and the normal control group $1(P>0.05)$.

\section{Gastric SOD activity}

SOD activity reflects the enzymatic antioxidative properties of tissues as it converts the highly reactive radicals $\left(\mathrm{O}_{2}^{-}\right)$into the less reactive $\mathrm{H}_{2} \mathrm{O}_{2}$ that can be destroyed by the CAT reaction. ${ }^{53}$ From Table 1, it can be noticed that treatment of rats with indomethacin significantly decreased SOD activity compared to the normal control rats $(P<0.05)$. Treatment with commercial FAM tablets significantly increased the SOD activity compared to the indomethacin-treated rat group $(P<0.05)$. On the other hand, treatment with the FAM beads significantly improved the SOD activity compared with group 2 $(P<0.05)$. Using a combination of FAM beads plus QRT for treatment of rats resulted in a significant increase in the SOD level compared with group $4(P<0.05)$. There was significant difference between the rats in this group and the rats in groups treated with either commercial FAM tablets or with FAM beads alone $(P<0.05)$. Again, it was noticed that there was nonsignificant difference between this group and rats of the normal control group $(P>0.05)$. This finding indicated the ability of the combination to renormalize SOD activity following indomethacin administration.

\section{Gastric CAT activity}

Inhibition of CAT activity enhances the generation of hydroxyl radicals as a result of prevention of antioxidant activity that leads to lipid peroxide formation. ${ }^{54}$ From Table 1, it could be noticed that the CAT activity was significantly decreased by indomethacin treatment when compared to its activity in the normal control group $(P<0.05)$. Treatment of animals with commercial FAM tablets significantly increased the CAT activity compared to the indomethacin-treated group $(P<0.05)$. Treatment of animals with the FAM beads significantly increased the CAT activity compared to the indomethacin-treated (group 2) or commercial FAM tablets-treated groups (group 3) $(P<0.05)$. Treatment of rats with a combination of beads plus QRT (group 5) significantly increased the CAT activity compared to the group treated with beads alone (group 4) $(P<0.05)$. The activity of CAT was found to be almost normalized upon treating animals with combination of FAM beads plus QRT. There was nonsignificant difference between CAT activities in rats of this group compared with that of the normal control group $(P>0.05)$. SOD and CAT have a major role in gastric oxidative/antioxidative balance. Reduction of both enzymes' activities in gastric mucosa as a result of ulcerogenic exposure leads to elevation in ROS levels. Accordingly, MDA levels increase. ${ }^{55}$ In our study, indomethacin induced inhibition of SOD and CAT activities with increase in the MDA concentration, which is in agreement with this finding.

From these results, the antioxidant activity of the prepared formula in gastric mucosal homogenates observed from decrease in LPO may be due to increase in SOD and CAT activities. It is well known that antioxidant activity is commonly related with gastroprotection and cytoprotection. ${ }^{56}$ Generally, the results obtained from this study showed the decrease of enzymatic and nonenzymatic oxidant parameters (compared to the control group that received indomethacin). The increase of antioxidant parameters observed in the gastric tissue of the animals that received FAM are in agreement with results of another study.$^{47}$ Our study demonstrated that oral treatment of indomethacin-induced gastric ulcer with the prepared FAM beads plus QRT for 15 consecutive days accelerates significantly the healing of chronic gastric ulcer in rats compared to either FAM commercial tablets or FAM beads alone.

\section{Conclusion}

Our study demonstrated that oral treatment of indomethacininduced gastric ulcer with the prepared FAM floating beads in combination with QRT-PVP for 15 consecutive days 
accelerates significantly the healing of chronic gastric ulcer in rats compared with either FAM commercial tablets or FAM beads alone. The combined drug formula lowered the enzymatic and nonenzymatic oxidant parameters, compared with the control group that received indomethacin. Biochemical assay and histopathological studies confirmed the anti-ulcer effect for both the formulated FAM beads and the combination treatment. Combination of the formulated FAM beads plus QRT-PVP promisingly revealed improved effectiveness in treatment of gastric ulcer compared with the commercial FAM tablets.

\section{Disclosure}

The authors report no conflicts of interest in this work.

\section{References}

1. Malfertheiner P, Chan FK, McColl KE. Peptic ulcer disease. Lancet. 2009;347:1449-1461.

2. Wu Y, Fassihi R. Stability of metronidazole, tetracycline $\mathrm{HCl}$ and famotidine alone and in combination. Int J Pharm. 2005;290:1-13.

3. Pilotto A, Franceschi M, Maggi S, Addante F, Sancarlo D. Optimal management of peptic ulcer disease in the elderly. Drugs Aging. 2010; 27:545-558.

4. Franceschi M, Di Mario F, Leandro G, Maggi S, Pilotto A. Acid-related disorders in the elderly. Best Pract Res Clin Gastroenterol. 2009;23: 839-848.

5. Huang JQ, Hunt RH. Pharmacological and Pharmacodynamic essentials of H(2)-receptor antagonists and proton pump inhibitors for the practising physician. Best Pract Res Clin Gastroenterol. 2001;15:355-370.

6. Lin JH. Pharmacokinetic and pharmacodynamics properties of histamine H2-receptor antagonists. Relationship between intrinsic potency and effective plasma concentrations. Clin Pharmacokinet. 1991;20:218-236.

7. Myers D. $\mathrm{H}_{2}$ Receptor Antagonists. J Exot Pet Med. 2006;15: $150-152$.

8. Okudaira K, Furuta T, Shirai N, Sugimoto M, Miura S. Concomitant dosing of famotidine with a triple therapy increases the cure rates of Helicobacter pylori infections in patients with the homozygous extensive metabolizer genotype of CYP2C19. Aliment Pharmacol Ther. 2005;21:491-497.

9. Razavi M, Nyamathulla S, Karimian H, Noordin MI. Novel swellable polymer of orchidaceae family for gastroretentive drug delivery of famotidine. Drug Des Devel Ther. 2014;8:1315-1329.

10. Murota K, Hotta A, Ido H, et al. Antioxidant capacity of albumin-bound quercetin metabolites after onion consumption in humans. J Med Invest. 2007;54:370-374.

11. Perez-Vizcaino F, Duarte J. Flavonols and cardiovascular disease. $M o l$ Aspects Med. 2010;31:478-494.

12. AhmedOA, Badr-Eldin SM, TawfikMK, AhmedTA, El-Say KM, BadrJM. Design and optimization of self-nanoemulsifying delivery system to enhance quercetin hepatoprotective activity in paracetamol-induced hepatotoxicity. J Pharm Sci. 2014;103:602-612.

13. Santos MR, Rodríguez-Gómez MJ, Justino GC, Charro N, Florencio MH, Mira L. Influence of the metabolic profile on the in vivo antioxidant activity of quercetin under a low dosage oral regimen in rats. Br J Pharmacol. 2008;153:1750-1761.

14. Borska S, Chmielewska M, Wysocka T, Drag-Zalesinska M, Zabel M, Dziegiel P. In vitro effect of quercetin on human gastric carcinoma: targeting cancer cells death and MDR. Food Chem Toxicol. 2012;50: 3375-3383.
15. Manach C, Texier O, Morand C, et al. Comparison of the bioavailability of quercetin and catechin in rats. Free Radic Biol Med. 1999;27: $1259-1266$.

16. Sánchez de Medina F, Gálvez J, González M, Zarzuelo A, Barrett KE. Effects of quercetin on epithelial chloride secretion. Life Sci. 1997;61: 2049-2055.

17. Sumbul S, Ahmad MA, Mohd A, Mohd A. Role of phenolic compounds in peptic ulcer: An overview. J Pharm Bioallied Sci. 2011;3: 361-367.

18. Ahmed OA, Badr-Eldin SM, Ahmed TA. Kinetic study of the in vitro release and stability of theophylline floating beads. Int J Pharm Pharm Sci. 2013;5:179-184.

19. Freitas FF, Fernandes HB, Piauilino CA, et al. Gastroprotective activity of Zanthoxylumrhoifolium Lam. In animal models. J Ethnopharmacol. 2011;137:700-708.

20. Sedlak J, Lindsay RH. Estimation of total, protein-bound, and nonprotein sulfhydryl groups in tissues with Ellmann's reagent. Anal Biochem. 1968;25:192-205

21. Panda V, Sonkamble M. Anti-ulcer activity of Ipomoea batatas tubers (sweet potato). Journal of Functional Foods in Health and Disease. 2012;2:48-61.

22. Polat B, Albayrak Y, Suleyman B, et al. Antiulcerative effect of dexmedetomidine on indomethacin-induced gastric ulcer in rats. Pharmacol Rep. 2011;63:518-526.

23. Sun Y, Oberley LW, Li Y. A simple method for clinical assay of superoxide dismutase. Clin Chem. 1988;34:497-500.

24. Sriamornsak P, Thirawong N, Puttipipatkhachorn S. Emulsion gel beads of calcium pectinate capable of floating on the gastric fluid: effect of some additives, hardening agent or coating on release behavior of metronidazole. Eur J Pharm Sci. 2005;24:363-373.

25. Rangaraj G, Kishore N, Dhanalekshmi UM, Raja MD, Senthil kumar C, Reddy PN. Design and study of formulation variables affecting drug loading and its release from Alginate beads. Journal of Pharmaceutical Sciences and Research. 2010;2:77-81.

26. Khonkarn R, Mankhetkorn S, Hennink WE, Okonogi S. PEG-OCL micelles for quercetin solubilisation and inhibition of cancer cell growth. Eur J Pharm Biopharm. 2011;79:268-275.

27. Zheng Y, Haworth IS, Zuo Z, Chow MS, Chow AH. Physicochemical and structural characterization of quercetin-beta-cyclodextrin complexes. J Pharm Sci. 2005;94:1079-1089.

28. Sri KV, Kondaiah A, Ratna JV, Annapurna A. Preparation and characterization of quercetin and rutin cyclodextrin inclusion complexes. Drug Dev Ind Pharm. 2007;33:245-253.

29. Sahoo NG, Kakran M, Shaal LA, et al. Preparation and characterization of quercetin nanocrystals. J Pharm Sci. 2011;100:2379-2390.

30. Kakran M, Sahoo NG, Li L, Judeh Z. Fabrication of quercetin nanoparticles by anti-solvent precipitation method for enhanced dissolution. Powder Technol. 2012;223:59-64.

31. Vicentini FT, Vaz MM, Fonseca YM, Bentley MV, Fonseca MJ. Characterization and stability study of a water-in-oil microemulsion incorporating quercetin. Drug Dev Ind Pharm. 2011;37:47-55.

32. Zheng Y, Chow AH. Production and characterization of a spray-dried hydroxypropyl-beta-cyclodextrin/quercetin complex. Drug Dev Ind Pharm. 2009;35:727-734.

33. Khaled KA, Mahrous GM. Comparative Study of the Dissolution and Physicochemical Characteristics of the Binary Systems of Quercetin with Polyethylene Glycol, Polyvinyl pyrrolidone, and Hydroxypropyl$\beta$-cyclodextrin. Saudi Pharm J. 2001;9:34-42.

34. Takeuchi K. Pathogenesis of NSAID-induced gastric damage: Importance of cyclooxygenase inhibition and gastric hypermotility. World J Gastroenterol. 2012;18:2147-2160.

35. Bandyopadhyay SK, Pakrashi SC, Pakrashi A. The role of antioxidant activity of Phyllanthus emblica fruits on prevention from indomethacin induced gastric ulcer. J Ethnopharmacol. 2000;70:171-176.

36. Sharma V, Rajani GP. Evaluation of Caesalpinia Linn. For anti-inflammatory and antiulcer activities. Indian J Pharmacol. 2011;43: 168-171. 
37. Bilici M, Ozturk C, Dursn H, et al. Protective effect of mirtazapine on indomethacin-induced ulcer in rats and its relationship with oxidant and antioxidant parameters. Dig Dis Sci. 2009;54:1868-1875.

38. Suleyman B, Halici Z, Odabasoglu F, Gocer F. The Effects of Lacidipine on Indomethacin Induced Ulcers in Rats. Int J Pharmacol. 2012;8(2): 115-121.

39. de Groot H, Rauen U. Tissue injury by reactive oxygen species and the protective effects of flavonoids. Fundam Clin Pharmacol. 1998;12: $249-255$.

40. Miura T, Muraoka S, Fujimoto Y. Lipid peroxidation induced by indomethacin with horseradish peroxidase and hydrogen peroxide: involvement of indomethacin radicals. Biochem Pharmacol. 2002;63: 2069-2074.

41. Suleyman H, Albayrak A, Bilici M, Cadirci E, Halici Z. Different mechanisms in formation and prevention of indomethacin-induced gastric ulcers. Inflammation. 2010;33:224-234.

42. Naito Y, Yoshikawa T, Yoshida N, Kondo M. Role of oxygen radical and lipid peroxidation in indomethacin-induced gastric mucosal injury. Dig Dis Sci. 1998;43:30S-34S.

43. El-Abhar HS. Coenzyme Q10: a novel gastroprotective effect via modulation of vascular permeability, prostaglandin $\mathrm{E}_{2}$, nitric oxide and redox status in indomethacin-induced gastric ulcer model. Eur J Pharmacol. 2010;649:314-319.

44. Kelkel M, Jacob C, Dicato M, Diederich M. Review: Potential of the dietary antioxidants resveratrol and curcumin in prevention and treatment of hematologic malignancies. Molecules. 2010;15:7035-7074.

45. Halliwell B, Whiteman M. Measuring reactive species and oxidative damage in vivo and in cell culture: how should you do it and what do the results mean? Br J Pharmacol. 2004;142:231-255.

46. Koc M, Imik H, Odabasoglu F. Gastroprotective and anti-oxidative properties of ascorbic acid on indomethacin-induced gastric injuries in rats. Biol Trace Elem Res. 2008;126:222-236.
47. Kisaoglu A, Ozoglu B, Cetyn N, Suleyman B, et al. The role of Alpha-2 Adrenergic Receptors in the Anti-ulcerative Activity of Famotidine and Omeprazole in Rats and its Relationship with Oxidant-antioxidant Parameters. Int J Pharmacol. 2011;7(6):682-689.

48. Cushnie TP, Lamb AJ. Antimicrobial activity of flavonoids. Int $J$ Antimicrob Agents. 2005;26:343-356.

49. Devi RS, Narayan S, Vani G, Shyamala Devi CS. Gastroprotective effect of Terminalia arjuna bark on diclofenac sodium induced gastric ulcer. Chem Biol Interact. 2007;167:71-83.

50. Bayir Y, Odabasoglu F, Cakir A, et al. The inhibition of gastric mucosal lesion, oxidative stress and neutrophil-infiltration in rats by the lichen constituent diffractaic acid. Phytomedicine. 2006;13:584-590.

51. Bombardier C. An evidence-based evaluation of the gastrointestinal safety of coxibs. Am J Cardiol. 2002;89:3D-9D.

52. Odabasoglu F, Cakir A, Suleyman H, et al. Gastroprotective and antioxidant effects of usnic acid on indomethacin-induced gastric ulcer in rats. J Ethnopharmacol. 2006;103:59-65.

53. Kwiecien S, Brzozowski T, Konturek SJ. Effects of reactive oxygen species action on gastric mucosa in various models of mucosal injuries. J Physiol Pharmacol. 2002;53:39-50.

54. Alvarez-Suarez JM, Dekanski D, Ristić S, et al. Strawberry polyphenols attenuate ethanol-induced gastric lesions in rats by activation of antioxidant enzymes and attenuation of MDA increase. PLoS One. 2011;6:e25878.

55. Gupta S, Kataria M, Gupta PK, Murganandan S, Yashroy RC. Protective role of extracts of neem seeds in diabetes caused by streptozotocin in rats. J Ethnopharmacol. 2004;90:185-189.

56. Zahorodnyǐ MI. [Effect of quercetin on sodium diclofenac-induced ulceration]. Lik Sprava. 2003;1:96-99. Ukrainian.
Drug Design, Development and Therapy

\section{Publish your work in this journal}

Drug Design, Development and Therapy is an international, peerreviewed open-access journal that spans the spectrum of drug design and development through to clinical applications. Clinical outcomes, patient safety, and programs for the development and effective, safe, and sustained use of medicines are a feature of the journal, which

\section{Dovepress}

has also been accepted for indexing on PubMed Central. The manuscript management system is completely online and includes a very quick and fair peer-review system, which is all easy to use. Visit http://www.dovepress.com/testimonials.php to read real quotes from published authors. 